\title{
A regression model for calculating the second dimension retention index in comprehensive two-dimensional gas chromatography time-of-flight mass spectrometry
}

Bing Wang ${ }^{\text {a,b, }}$, Hao Shen ${ }^{\mathrm{a}}$, Aiqin Fang ${ }^{\mathrm{d}}$, De-shuang Huang ${ }^{\mathrm{a}}$, Changjun Jiang ${ }^{\mathrm{a}}$, Jun Zhang $^{\mathrm{e}, *}$, Peng Chen ${ }^{\mathrm{f}, *}$

${ }^{\mathrm{a} C}$ College of Electronics and Information Engineering, Tongji University, Shanghai 201804, china

${ }^{\mathrm{b}}$ The Advanced Research Institute of Intelligent Sensing Network, Tongji University, Shanghai 201804, china

${ }^{\mathrm{c}}$ The Key Laboratory of Embedded System and Service Computing, Tongji University, Shanghai 201804, china

${ }^{\mathrm{d}}$ Bioproduct and Biosystem Engineering, University of Minnesota, Twin Cities, MN 55108, USA

${ }^{\mathrm{e}}$ College of Electrical Engineering and Automation, Anhui University, Hefei, Anhui, China

${ }^{\mathrm{f}}$ Institute of Health Sciences, Anhui University, Hefei, Anhui, China

* Corresponding author. Tel.: +861381760 5997.

E-mail address: wangbing@ustc.edu (B. Wang). 


\section{ABSTRACT}

Comprehensive two-dimensional gas chromatography time-of-flight mass spectrometry (GC×GC/TOF-MS) system has become a key analytical technology in high-throughput analysis. Retention index has been approved to be helpful for compound identification in one-dimensional gas chromatography, which is also true for two-dimensional gas chromatography. In this work, a novel regression model was proposed for calculating the second dimension retention index of target components where $n$-alkanes were used as reference compounds. This model was developed to depict the relationship among adjusted second dimension retention time, temperature of the second dimension column and carbon number of $n$-alkanes by an exponential nonlinear function with only five parameters. Three different criteria were introduced to find the optimal values of parameters. The performance of this model was evaluated using experimental data of $n$-alkanes $\left(\mathrm{C}_{7}-\mathrm{C}_{31}\right)$ at 24 temperatures which can cover all 0-6 s adjusted retention time area. The experimental results show that the mean relative error between predicted adjusted retention time and experimental data of $n$-alkanes was only $2 \%$. Furthermore, our proposed model demonstrates a good extrapolation capability for predicting adjusted retention time of target compounds which located out of the range of the reference compounds in the second dimension adjusted retention time space. Our work shows the deviation was less than 9 retention index units (iu) while the number of alkanes were added up to 5. The performance of our proposed model has also been demonstrated by analyzing a mixture of compounds in temperature programmed experiments. 
Keywords:

GC $\times$ GC/TOF-MS

Regression model

Retention time

Retention index

Second dimension retention time 


\section{Introduction}

Mass spectrometry (MS) coupled with chromatographic separation techniques has become a key analytical technology in high-throughput analysis of small molecules [1]. In 1990s, Liu and Phillips invented comprehensive two-dimensional gas chromatography $(\mathrm{GC} \times \mathrm{GC})$ with a modular between two distinctly different capillary columns of different polarities [2]. The first dimension column has a length of 15-30m, while the second dimension column is only $0.5-2 \mathrm{~m}$ [3-5], which means the separation in the second dimension column is so fast that it can be treated as in pseudo-isothermal conditions [6]. Compared with one-dimensional gas chromatography (1-D GC), GC $\times \mathrm{GC}$ has great improvement in column capacity and compounds separation $[3,7]$. In recent years, comprehensive two-dimensional gas chromatography/time-of-flight mass spectrometry (GC $\times \mathrm{GC} / \mathrm{TOF}-\mathrm{MS})$ has been extensively applied in analyzing complex mixtures such as oil, food, drug, metabolomical and biological samples [8-13].

Currently, compound identification through searching spectrum library is the most popular method in analyzing experimental spectra generated from $\mathrm{GC} \times \mathrm{GC} / \mathrm{TOF}-\mathrm{MS}$ system [14-16]. However, this method produces many false-positive identifications, especially for complex mixtures analysis. For example, two compounds with similar structure may have similar fragment of mass spectra, and homologous compounds may have very similar mass spectra too [17]. Therefore, more information, such as separation status of molecule, should be involved to increase identification confidence. Retention index (RI), a standardized retention time which calculated by $n$-alkanes in 
1-D GC, are widely used to aid compound identification [18, 19]. For this purpose, lots of retention index libraries have been developed for 1-D Gas Chromatography [15, $16,20-23]$.

In addition, there are also a few works focusing on 2-D retention index for GC $\times$ GC/TOF-MS. Beens et al. first constructed a 2-D retention map with continuous injection of $n$-dodecane, and mentioned three strategies in estimating the second column hold-up time $\left({ }^{2} t_{M}\right)$ [24], and used five alkanes $\left(\mathrm{C}_{9}-\mathrm{C}_{13}\right)$ as reference compounds later [25]. Western and Marriott made further study with different injection methods, initial temperatures, temperature ramp rate, and reference compounds. They also calculated the second dimension retention index with 2-D retention map to identify compounds $[6,26]$. Zhu et al. used a similar strategy as Western and Marriott to identify the unknown compounds in cigarette essential oil [27]. Pang et al. made a change of columns set order to identify unknown compounds in tobacco leaves and adopted regression function to extend the coverage of 2-D retention map [28]. Bieri and Marriott proposed the solid-phase microextraction (SPME) method [29] and a double injection system to study the elution range covered by reference compounds $\mathrm{C}_{10}-\mathrm{C}_{21}$ [30]. Zhao et al. proposed a novel function to construct an adjusted second dimension retention time map [31]. More information about retention indices in $\mathrm{GC} \times \mathrm{GC}$ can be found in Mühlen and Marriott's review paper [32]. However, the application of 2-D RI based on $n$-alkanes was limited in GC $\times$ GC/TOF-MS because of the polarity of the second column. Many polar compounds experimentally eluted after reference compounds $\mathrm{C}_{9}-\mathrm{C}_{22}$ in the second 
dimension column, which exceed the coverage of 2-D reference map [26]. Therefore, new calculation methods which can expand the coverage of second dimension RI are needed. Another limitation of previous studies is that the calculation models are very complicated. For example, there are ten parameters have to be determined in a polynomial adjusted retention time fitting model proposed by Zhao et al., which raises the computational difficulty and the likelihood of falling into overfitting problem. To overcome the coverage and computational complexity limitations, a practical method with better extrapolation capability for calculating the second dimension retention index is needed.

To meet these needs, this work proposed a novel computational model to calculate the second dimension retention index $\left({ }^{2} I\right)$ with better robustness, which even works for some compounds located out of the range of reference compounds in the adjusted second dimension retention time map. Generally, ${ }^{2} I$ can be calculated with Kováts Retention Index function even in temperature programmed experiments because the second dimension column can be seen as pseudo-isothermal $[6,33,34]$. In our work, a new simple second dimension adjusted retention time-second column temperature $\left({ }^{2} t_{R}-{ }^{2} T_{e}\right)$ function was developed to fit adjusted experimental retention times of the reference $n$-alkanes based on an adjusted second dimension retention time map which was constructed by using experimental data from the $\mathrm{GC} \times \mathrm{GC} / \mathrm{TOF}-\mathrm{MS}$ runs at various second dimension temperatures with $n$-alkanes as reference compounds. In this work, three different criteria were introduced to find optimal parameters of the regression function. It can be found from our experiments that with the proposed 
${ }^{2} t_{R},{ }^{2} T_{e}$ function and criteria, adjusted retention time of $n$-alkanes in the second dimension can be predicted more accurately. In addition, retention index of target compounds, no matter eluted before or after reference $n$-alkanes, can also be calculated. A standard mixture of different compounds and reference $n$-alkanes were used to evaluate the performance of our proposed model.

\section{Experimental Materials}

\subsection{Reagents}

Dichloromethane and $\mathrm{C}_{7}-\mathrm{C}_{40} \quad n$-alkane mixture were purchased from Sigma-Aldrich (St. Louis, MO). 8270 MegaMix (76 components) were obtained from Restek Corp. (Bellefonte, PA). Alkane mixture was prepared in dichloromethane with a concentration $2.5 \mu \mathrm{g} / \mathrm{mL}$ per compound.

\subsection{Instrumentation and operational conditions}

An Agilent Technologies 6890 gas chromatograph (Santa Clara, CA) was hyphenated to a LECO Pegasus III TOF MS (St. Joseph, MI). The instrument was outfitted with the commercially available Gerstel MPS2 autosampler (Baltimore, MD)

In the $\mathrm{GC} \times \mathrm{GC}$ configuration, a $30 \mathrm{~m} \times 0.25 \mathrm{~mm}$ ID $\times 0.25 \mu \mathrm{m}$ film thickness, DB-5ms (Agilent Technologies, Santa Clara, CA) GC capillary column, was used as the primary column for the $\mathrm{GC} \times \mathrm{GC} / \mathrm{TOF}-\mathrm{MS}$ analysis. A second column $1.8 \mathrm{~m} \times$ $0.10 \mathrm{~mm}$ ID $\times 0.10 \mu \mathrm{m}$ film thickness, DB-17 (Agilent Technologies, Santa Clara, CA), was placed inside the LECO secondary GC oven after the thermal modulator. The helium carrier gas flow rate was set to $1.0 \mathrm{~mL} \mathrm{~min}^{-1}$ at a corrected constant flow 
via pressure ramps. A $2 \mu \mathrm{L}$ split liquid injections (the split ratio was set at 100:1) were made with the injection port temperature set at $260{ }^{\circ} \mathrm{C}$. The thermal modulator was set to $+20{ }^{\circ} \mathrm{C}$ relative to the primary oven and the second oven was $+5{ }^{\circ} \mathrm{C}$ to the primary oven. A modulation time of $6 \mathrm{sec}$ was used for temperature programmed experiments and $9 \mathrm{sec}$ for isothermal experiments. The MS mass range was $\mathrm{m} / \mathrm{z} 45-800$ with an acquisition rate of 200 spectra per second. The ion source chamber was set at $230{ }^{\circ} \mathrm{C}$ with the MS transfer line temperature set at $225^{\circ} \mathrm{C}$ and the detector voltage was 1700 $\mathrm{V}$ with an electron energy of $70 \mathrm{eV}$. In isothermal experiments, alkane mixture was analyzed with first column temperature constantly at $60,70,80,90,100,110,120,130$, $140,150,160,170,180,190,200,210,220,230,240,250,260,265,270$ and $280{ }^{\circ} \mathrm{C}$, respectively. The total analysis time was $60 \mathrm{~min}$ for each run. In temperature programmed experiments, the analysis of alkane and MegaMix mixture was performed with primary column initial temperature at 60, 80, 100, 120, 140, and 160 ${ }^{\circ} \mathrm{C}$ for $0.5 \mathrm{~min}$, respectively. Then the column temperature ramped to $280{ }^{\circ} \mathrm{C}$ at $5{ }^{\circ} \mathrm{C}$ /min with a hold time $20 \mathrm{~min}$.

\subsection{Raw data reduction}

The LECO ChromaTOF software (version 4.21), equipped with the National Institute of Standards and Technology (NIST) MS database (NIST MS Search 2.0, NIST/EPA/NIH Mass Spectral Library; NIST 2002), was used for instrument control, spectrum deconvolution, and compound identification. By manually visualizing the raw data, the peak width on the first dimension column was estimated to range from 2 to 3 modulation periods, while ranges from $0.2-0.5 \mathrm{~s}$ on the second dimension column. 
Manufacturer recommended parameters for ChromaTOF were used to reduce the raw instrument data into a compound peak list, and were set as follows: baseline offset = 0.5 ; smoothing $=$ auto; peak width in first dimension $=6 \mathrm{~s}$; peak width in the second dimension $=0.1 \mathrm{~s} ;$ signal-to-noise ratio $=100 ;$ match required to combine peaks $=500 ;$ R.T. shift $=0.08 \mathrm{~s} ;$ minimum forward similarity match $=600$. The true peak spectrum was also exported as part of the information for each peak in absolute format of intensity values.

\section{Method}

\subsection{Basic model}

The separation in the second dimension column can be regarded as in pseudo-isothermal condition in temperature programmed experiments because the length of the second dimension column is very short. Therefore, some methods reported for retention time calculation in 1-D GC can still be applied in GC×GC. Koo et al. investigated six computational models and found the most robust approaches was the one developed by J.E. Quintanilla-Lopez et al. [33, 35], which is also the basic model in our study:

${ }^{2} t_{R}=p_{1}+\exp \left(p_{2} \times N+p_{3}\right)$

where ${ }^{2} t_{R}$ is the second dimension retention time of $n$-alkanes, $p_{i}$ are coefficients of the model, and $N$ is the carbon number of a $n$-alkane.

\subsection{Hold-up time}

As the head pressure was set constant as 65 psi in operating conditions of all 
experiments, the hold-up time in the second dimension $\left({ }^{2} t_{M}\right)$ is only related to the temperature of the second dimension column $\left({ }^{2} T_{e}\right)$. Zhao et al. proposed a function to calculate ${ }^{2} t_{M}$ value based on ${ }^{2} T_{e}$ with a reliable fitting accuracy $R^{2}=0.9973$ [31]. Zhao's ${ }^{2} t_{M}$ function has been adopted in this work because we use the same experimental configuration and data source. Therefore the second dimension retention time $\left({ }^{2} t_{R}\right)$ could be transformed to the second dimension adjusted retention time $\left({ }^{2} t_{R}{ }^{\prime}\right)$ by minus ${ }^{2} t_{M}$.as follows:

${ }^{2} t_{M}=0.000004 \times\left({ }^{2} T_{e}\right)^{2}-0.0025 \times\left({ }^{2} T_{e}\right)+1.6184$

where ${ }^{2} t_{M}$ is the fitted hold-up time in the second dimension and ${ }^{2} T_{e}$ is the second dimension column temperature in ${ }^{\circ} \mathrm{C}$.

\subsection{Construction of the ${ }^{2} t_{R}{ }^{\prime}{ }^{2} T_{e}$ model}

By the above processes, it can be found that the second dimension retention time of $n$-alkanes now varies only with temperature of the second dimension and carbon number of alkanes. Because Koo et al. had demonstrated the exponential model outperform polynomial functions in calculating ${ }^{2} t_{R}$ [33], the ${ }^{2} t_{R}{ }^{2}-{ }^{2} T_{e}$ function in our work can be constructed based on Eq. (1) as follows:

${ }^{2} t_{R}{ }^{\prime}=\exp \left(a\left({ }^{2} T_{e}\right) \times N+b\left({ }^{2} T_{e}\right)\right)$

where ${ }^{2} t_{R}$ ' is the adjusted second dimension retention time of $n$-alkanes; ${ }^{2} T_{e}$ is the temperature of the second column; $N$ is the number of carbon atom; $a\left({ }^{2} T_{e}\right)$ and $b\left({ }^{2} T_{e}\right)$ both are dependent variables of ${ }^{2} T_{e}$, whose values could be calculated using the basic model at different temperature situation.

3.4. Three criteria in calculation of coefficients 
It can be seen that for reference $n$-alkanes the adjusted retention time, where hold-time has been subtracted from the whole retention time, is dependent on the value of function $a\left({ }^{2} T_{e}\right)$ and $b\left({ }^{2} T_{e}\right)$. Therefore the problem now comes to how to determine the form of these two ${ }^{2} T_{e}$-based functions. In our work, $a\left({ }^{2} T_{e}\right)$ and $b\left({ }^{2} T_{e}\right)$ were discussed separately. Three criteria, i.e., minimum square error (MSE), Minimum square relative error (MSRE) and Combination of both of them (Comb), were adopted to evaluate the performance of ${ }^{2} t_{R}{ }^{2} T_{e}$ function with different parameters. Here the nonlinear least squares method and Gauss-Newton gradient descent method were used to calculate the values of parameters [36, 37].

Three criteria are defined as follows:

$$
\begin{aligned}
& \text { MSE }:\left|{ }^{2} t_{R}{ }^{\prime}{ }_{p}-{ }^{2} t_{R}{ }^{\prime}\right|^{2} \\
& \text { MSRE }:\left|\frac{{ }^{2} t_{R}{ }^{\prime}-{ }^{2} t_{R}{ }^{\prime}{ }_{p}}{{ }^{2} t_{R}{ }^{\prime}}\right|^{2} \\
& \text { Comb }:\left|{ }^{2} t_{R}{ }^{\prime}-{ }^{2} t_{R}{ }^{\prime}{ }_{p}\right|\left|\frac{{ }^{2} t_{R}{ }^{\prime}-{ }^{2} t_{R}{ }^{\prime}{ }_{p}}{{ }^{2} t_{R}{ }^{\prime}}\right|
\end{aligned}
$$

where ${ }^{2} t_{R}{ }^{\prime}$ and ${ }^{2} t_{R}$ ' are the predicted and true value of the adjusted retention time of $n$-alkanes in the second dimension column.

\subsection{Evaluating the performance of the proposed model}

In the $\mathrm{GC} \times \mathrm{GC} / \mathrm{TOF}-\mathrm{MS}$ system, the Kováts retention index function can be used to calculate second dimension retention index values of target compounds because of isothermal or pseudo-isothermal condition in the second dimension column [6], which can be defined as follows [34]:

$$
I=100 \times\left[n+\frac{\log \left(t_{R}{ }^{\prime}(\text { unknown })\right)-\log \left(t_{R}{ }^{\prime}(n)\right)}{\log \left(t_{R}{ }^{\prime}(n+1)\right)-\log \left(t_{R}{ }^{\prime}(n)\right)}\right]
$$


where $I$ is the retention index and $t_{R}{ }^{\prime}($ unknown) is the adjusted retention time of the unknown compound in the second dimension; $t_{R}{ }^{\prime}(n)$ and $t_{R}{ }^{\prime}(n+1)$ are the adjusted retention time of two continuous $n$-alkanes between which the target compound eluted off the second dimension column.

In order to evaluate the performance of the proposed ${ }^{2} t_{R}{ }^{\prime}-{ }^{2} T_{e}$ regression model, three measures, i.e., the mean absolute error (MAE), the root mean square error (RMSE) and the mean relative error (MRE), were adopted to depict the distribution of the deviation between the predicted value and true value for assessing the fitting capability.

$$
\begin{aligned}
& M A E=\frac{1}{N} \sum_{i=1}^{N}\left|t_{R}^{2} t^{\prime}{ }^{i}{ }^{2} t_{R}{ }^{i}\right| \\
& R M S E=\sqrt{\frac{1}{N} \sum_{i=1}^{N}\left({ }^{2} t_{R}^{\prime}{ }^{i}-t_{R}{ }^{i}\right)^{2}} \\
& M R E=\frac{1}{N} \sum_{i=1}^{N}\left|\frac{t_{R}{ }^{\prime} p^{i}-t_{R}{ }^{i^{i}}}{t_{R}{ }^{i^{i}}}\right|
\end{aligned}
$$

where $N$ is the number of all $\mathrm{C}_{\mathrm{n}}$-alkanes' adjusted retention time data; ${ }^{2} t_{R}{ }^{i}$ and ${ }^{2} t_{R}{ }^{i}$ are the predicted and true value of the adjusted retention time of the $i$ th $\mathrm{C}_{\mathrm{n}}$-alkane in the second dimension.

In this work, the $\mathrm{C}_{\mathrm{n}}$-alkanes' adjusted retention time were firstly predicted and compared with the empirical data where the second dimension hold-up time had been corrected using Eq. (2). The predicted retention index of $\mathrm{C}_{\mathrm{n}}$-alkanes was then calculated with fitted ${ }^{2} t_{R}{ }^{\prime}-T_{e}$ curves of $\mathrm{C}_{\mathrm{n}-1}$-alkanes and $\mathrm{C}_{\mathrm{n}+1}$-alkanes in each temperature. MAE and RMSE were adopted to evaluate the method in both fitting and 
predicting function. It should be noted that the full dataset was used when we assessed the fitting capability of our method, and it was partitioned as training set and test set in evaluating prediction power.

Furthermore, a standard mixture of different compounds called MegaMix was used to assess the accuracy of our proposed method in converting second dimension retention time to retention index for components identification. As the data of MegaMix were collected at different temperatures, the standard deviation (STD) and the mean standard deviation $(\overline{S T D})$ of all compounds were calculated as follows:

$$
\begin{aligned}
& S T D_{i}=\sqrt{\frac{1}{M_{i}-1} \sum_{j=1}^{M_{i}}\left(2 I_{j}^{i}-\frac{1}{M_{i}} \sum_{k=1}^{M_{i}}{ }^{2} I_{j}^{i}\right)^{2}} \\
& \overline{S T D}=\frac{1}{N} \sum_{i=1}^{N} S T D_{i}
\end{aligned}
$$

where ${ }^{2} I_{j}^{i}$ is the retention index of the $i$ th compound at the $j$ th temperature; $M_{i}$ is the number of the retention index values of the $i$ th compound; $N$ is the number of compounds in the MegaMix.

\section{Results and discussion}

In this study, an adjusted second dimension retention time map was constructed to cover the whole $0-6 \mathrm{~s}$ adjusted retention time domain in the second dimension, where $n$-alkanes $\left(\mathrm{C}_{7}-\mathrm{C}_{31}\right)$ were introduced as reference compounds in GC/MS system. Then a simple and practical ${ }^{2} t_{R}-{ }^{2} T_{e}$ regression function was proposed to predict adjusted retention time and retention index in the second dimension column. The good extrapolation capability of our regression model allows the extension of the coverage 
of reference $n$-alkanes to the whole second dimension retention time area, even places that were not covered by the experimental data. Analysis of a mixture of $n$-alkanes and Megamix was performed on the $\mathrm{GC} \times \mathrm{GC} / \mathrm{TOF}-\mathrm{MS}$ system for further evaluation.

\subsection{Determination of ${ }^{2} t_{R}{ }^{\prime}-{ }^{2} T_{e}$ regression model}

In our ${ }^{2} t_{R}{ }^{\prime}-{ }^{2} T_{e}$ regression model, there are two variables in Eq. (3), i.e., $a\left({ }^{2} T_{e}\right)$ and $b\left({ }^{2} T_{e}\right)$, which need to be defined. It can be found that both of them are dependent on the temperature variable ${ }^{2} T_{e}$. Fig 1 shows the distribution of $a\left({ }^{2} T_{e}\right)$, and it can be found that its value descends regularly with the increase of the temperature ${ }^{2} T_{e}$, and the changes can be fitted using an experiential regression function as follow:

$$
\ln \left(a\left({ }^{2} T_{e}\right)\right)=p_{1} \times{ }^{2} T_{e}+p_{2}
$$

where $p_{1}, p_{2}$ are parameters of the fitting function which can be calculated by using MSE criterion, and the best fitting can be got when $p_{1}=-0.00585879$, and $p_{2}=$ 0.06050222 .

$b\left({ }^{2} T_{e}\right)$ can be fitted using linear and nonlinear models based on the distribution of points. By comparison of the fitting effect of linear model (Fig. 2) and nonlinear model (Fig. 3), the nonlinear model was adopted in this work. Because $b\left({ }^{2} T_{e}\right)$ are negative, the nonlinear regression function is exponentially fitted as follows:

$$
\ln \left(b\left({ }^{2} T_{e}\right)+p_{1}\right)=\left(p_{2}-{ }^{2} T_{e}\right) \times p_{3}+p_{4}
$$

where $p_{i}$ are parameters of the fitting function. Therefore, $b\left({ }^{2} T_{e}\right)$ can be calculated as follows:

$$
b\left({ }^{2} T_{e}\right)=\exp \left({ }^{2} T_{e} \times p_{1}+p_{2}\right)+p_{3}
$$

where $p_{i}$ are parameters of the fitting function, which can be determined using MSE 
criterion, nonlinear regression and Gauss-Newton gradient descent method [35]. Fig. 3 shows the fitting curve.

By combining Eq. $(3,13,15)$, a multiple fitting model of the ${ }^{2} t_{R}{ }^{\prime}-T_{e}$ function can be constructed as follows:

$$
{ }^{2} t_{R}{ }^{\prime}=\exp \left(\exp \left(p_{1} \times{ }^{2} T_{e}+p_{2}\right) \times N+\exp \left(p_{3} \times{ }^{2} T_{e}+p_{4}\right)+p_{5}\right)
$$

where ${ }^{2} t_{R}$ ' is the adjusted second dimension retention time; $p_{i}$ are parameters of the fitting function; $N$ is the carbon number of a $n$-alkane. And the initial values of the parameters (Initial Value) can be obtained while the fitting function was established: $p_{1}=-0.00585879 ; p_{2}=0.06050222 ; p_{3}=0.00307490 ; p_{4}=-0.09757954 ; p_{5}=$ $-7.26213418$

4.2. The adjusted second dimension retention time map

Based on experimental data with $\mathrm{C}_{\mathrm{n}}$-alkanes $\left(\mathrm{C}_{7}-\mathrm{C}_{31}\right)$ as reference compounds, the adjusted second dimension retention time map can be constructed as shown in Fig. 4. With the above initial values of coefficients, the proposed ${ }^{2} t_{R}-{ }^{2} T_{e}$ function was applied to fit all of experimental data. Within the adjusted retention time area covered by reference $n$-alkanes in the adjusted second dimension retention time map, the retention index of target compounds who located in that region can be calculated with Kováts function Eq. (7). While we extended the coverage with our method by increasing the carbon number of $n$-alkanes and setting target temperature, retention indices of more compounds can be calculated.

\subsection{The performance of the proposed regression model}

Our proposed model showed not only the relationship between adjusted retention 
time and carbon number of $\mathrm{C}_{\mathrm{n}}$-alkanes as Kováts [34], but also the relationship between adjusted retention time and ${ }^{2} T_{e}$. With this function, the limitation of the application of retention index with reference $n$-alkanes in the polar second dimension was overcome by increasing carbon number $N$.

\subsubsection{Coefficients under different criteria}

The parameters in the proposed ${ }^{2} t_{R}{ }^{\prime}-T_{e}$ function were determined by adjusted retention time data, temperature of the second dimension column and carbon number of $n$-alkanes. In order to analyze the performance of proposed ${ }^{2} t_{R}{ }^{2}-T_{e}$ function, all coefficients were calculated under different criteria Eq. (4-6) with initial values of parameters and all of empirical data.

The adjusted retention time of all $\mathrm{C}_{n}$-alkanes $\left(\mathrm{C}_{7}-\mathrm{C}_{31}\right)$ were firstly predicted with the full dataset using the proposed criteria and Gauss-Newton gradient descent method. The predicted ${ }^{2} t_{R}$ ' was then computed by the proposed ${ }^{2} t_{R}{ }^{2}-T_{e}$ function. Table 1 displayed the results under three different criteria, i.e., MSE, MSRE and Comb, to show the deviations featured by MAE, RMSE and MRE between the predicted and experimental values on the full dataset.

For each $\mathrm{C}_{\mathrm{n}}$-alkanes, its retention index can be predicted by the fitted curves of $\mathrm{C}_{\mathrm{n}-1}$-alkanes and $\mathrm{C}_{\mathrm{n}+1}$-alkanes at different temperatures of the second dimension column. MAE and RMSE were then computed to show the deviation between the predicted and the standard retention index value on the full dataset shown in Table 2.

\subsubsection{The interpolation and extrapolation capability of the proposed method}

It should be noted that there are always many compounds cannot be covered by 
region of reference $n$-alkanes on the adjusted second dimension retention time map, which causes a problem that how to evaluate the performance of the regression model when molecules eluted out of the coverage area of $n$-alkanes. It is well known that regression models cannot guarantee the extrapolation performance even though they work well for the interpolation. For example, a good regression model may produce an accurate value for predicting adjusted retention time of $\mathrm{C}_{\mathrm{i}}$-alkane based on the experimental retention times of $\mathrm{C}_{\mathrm{i}-1}$-alkane and $\mathrm{C}_{\mathrm{i}+1}$-alkane. However, it does not means it can also make good predictions for $\mathrm{C}_{\mathrm{i}-2^{-}}$or $\mathrm{C}_{\mathrm{i}+2^{-}}$-alkane.

In order to evaluate the extensional capability of the proposed function and criteria, we reconstruct the predicted ${ }^{2} t_{R}-{ }^{2} T_{e}$ curves of $\mathrm{C}_{7}-\mathrm{C}_{31} n$-alkanes based on a training set only contains the isothermal data of $\mathrm{C}_{7}-\mathrm{C}_{26} n$-alkanes. The parameters were calculated with Eq. (16) under Eq. (4-6), respectively. Based on our proposed ${ }^{2} t_{R}{ }^{\prime}-{ }^{2} T_{e}$ function and coefficients, it is easy to make an extension of the coverage of retention $n$-alkanes in the second dimension time space by setting the carbon number $\mathrm{N}$ and the temperature ${ }^{2} T_{e}$.

Fig. 5 shows the adjusted second dimension retention time maps under criterion MSE, where Fig. 5(a) is constructed on the original full experimental $C_{7}-C_{31}$ dataset, and Fig. 5(b) is on the predicted values of $\mathrm{C}_{7}-\mathrm{C}_{31}$ based on experimental $\mathrm{C}_{7}-\mathrm{C}_{26}$ dataset. It can be found that the deviation of the predicted curve increased with the carbon number when ${ }^{2} T_{e}$ is higher. However, even for $\mathrm{C}_{31}$, the compound with the biggest carbon number in our work, the deviation is very small. Especially, from Fig. 5(b), it can be found that our proposed function has good extrapolation capability. 
When other criteria, i.e., MSRE and Comb, were adopted, our proposed function shows better extrapolation capability than MSE did, which can be found from Fig. S1 and Fig. S2 in supplementary file.

Table 3 shows the comparison of the MAE of ${ }^{2} I$ between standard values (defined in Kováts Retention Index) and predicted values calculated by different methods mentioned in Sec 3.4. It can be seen that MAE is always less than 9.20 iu with MSRE and Comb criteria, which means the value of $\mathrm{C}_{\mathrm{n}}$-alkanes' adjusted retention time can be predicted very well. In addition, the difference of predicted values based on $\mathrm{C}_{7}-\mathrm{C}_{31}$ and $\mathrm{C}_{7}-\mathrm{C}_{26}$ dataset is small, especially for MSRE and Comb criteria, which demonstrates the robustness of our proposed model.

It also can be found from Table 3 that our proposed model have better prediction performance in comparison with Zhao's method. For all three proposed criteria, the MAE of predicted ${ }^{2} I$ of $\mathrm{C}_{27}-\mathrm{C}_{31}$ did not increase much with the carbon number, which indicates that our method can predict more $\mathrm{C}_{\mathrm{n}}$-alkanes' adjusted retention time by increasing the carbon number. Therefore, the proposed method not only has better performance in interpolation, but also a great improvement in extrapolation.

In this work, the fitting equations are based on a serial isothermal experiments at 24 different temperatures. To further evaluate the performance of our proposed method more reasonably and avoid potential overfitting problem, we implemented the approach using only a small part of the original data set, i.e., data from one fourth temperatures of our original experiments. We generated four new datasets D1, D2, D3 and D4 from the original full data set, where D1 came from a sub-temperature set (65, 
$\left.105,145,185,225,265^{\circ} \mathrm{C}\right), \mathrm{D} 2$ from $\left(75,115,155,195,235,270{ }^{\circ} \mathrm{C}\right), \mathrm{D} 3$ from $(85$, $\left.125,165,205,245,275^{\circ} \mathrm{C}\right)$ and $\mathrm{D} 4$ from $\left(95,135,175,215,255,285^{\circ} \mathrm{C}\right)$. Based on each of the four datasets, we reconstructed the predicted ${ }^{2} t_{R}{ }^{2}-T_{e}$ curves when ${ }^{2} T_{e}$ changed within a range of $65-285^{\circ} \mathrm{C}$, where the parameters were calculated with Eq. (16) under Eq. (4-6), like what we did before. Table 4 shows the comparison of the MAE and RMSE of ${ }^{2} I$ with the proposed ${ }^{2} t_{R}{ }^{\prime}-{ }^{2} T_{e}$ function, different criteria and training sets, while the full dataset had been taken as test data. Compared with Table 2, although the number of temperatures used here was reduced from 24 to 6 , the change of the MAE and RMSE of ${ }^{2} I$ was very small. The similar accuracy further demonstrated the robustness of our proposed method.

\subsection{Analysis of a standard mixture}

To further assess the performance of our proposed model, MegaMix, a standard mixture of $76+$ compounds, has been analyzed in this work. Mixed with $n$-alkanes, MegaMix was analyzed in $\mathrm{GC} \times \mathrm{GC} / \mathrm{TOF}-\mathrm{MS}$ system with a temperature programmed mode at 6 different initial temperatures. Among the MegaMix, 47 compounds was identified from experiments as listed in Table S1.

In order to convert the adjusted retention time of identified compounds of MegaMix to the retention index, the proposed ${ }^{2} t_{R}{ }^{2}{ }^{2} T_{e}$ function was firstly adopted to calculate the adjusted retention time of $\mathrm{C}_{\mathrm{n}}$-alkanes, which were used as reference compounds. Then the second dimension retention index was calculated with Kováts function Eq. (7) [34]. The values of parameters were obtained in Sec 4.1 while the adjusted second dimension retention time maps were constructed using the original 
full dataset. The standard deviation $(S T D)$ of the second dimension retention index of identified 47 compounds ranged from 1-10 iu (for Initial Value, MSRE, Comb) and 1-11 iu (for MSE), respectively, which are smaller than the performance of Zhao's model, i.e., 1-12 iu [31]. When the MSRE criterion was chosen, only one compound has the $S T D$ of ${ }^{2} I$ of $10 \mathrm{iu}$, and all of others less than $10 \mathrm{iu}$. The mean standard deviation ( $\overline{S T D}$ ) were 4.89 for Initial Value, 5.06 for MSRE, 5.13 for Comb, and 5.45 for MSE. These results shows that, in the GC $\times \mathrm{GC} / \mathrm{TOF}-\mathrm{MS}$ system, ${ }^{2} I$ of target compounds in complex mixtures can be obtained by converting adjusted retention time values of these compounds using the proposed ${ }^{2} t_{R}-{ }^{2} T_{e}$ model in this work. Moreover, the stability of the calculated second dimension retention index outperforms the report in the previous similar work with only half number of coefficients, especially when reasonable criterion was chosen [31].

\section{Conclusion}

As in 1-D GC, the homologue $n$-alkanes were chosen as reference compounds to enhance the identification confidence of target compounds in GC×GC/TOF-MS system. But compared to 1 -D GC, the coverage of reference $n$-alkanes was limited because of the restriction of the polarity of the second dimension column. To overcome this limitation, a novel ${ }^{2} t_{R}{ }^{\prime}-{ }^{2} T_{e}$ model was developed to depict the relationship of adjusted retention time, carbon number of $\mathrm{C}_{\mathrm{n}}$-alkanes and ${ }^{2} T_{e}$ based on a dataset from 24 isothermal experiments. In our proposed ${ }^{2} t_{R}{ }^{2}{ }^{2} T_{e}$ regression model, fewer coefficients were needed, which decreased the complexity of the function and 
the calculation of parameters. Here, three criteria were also introduced to find the optimal values of model parameters and MSRE criterion usually achieved best performance.

An adjusted second dimension retention time map covered all the 0-6 s adjusted retention time area was constructed based on the empirical data, which was very helpful for the calculation of retention index of target compounds located within that region. In addition, our proposed ${ }^{2} t_{R}{ }^{,}-T_{e}$ model can greatly extended the coverage of reference $n$-alkanes in the adjusted second dimension retention time map by setting the carbon number and ${ }^{2} T_{e}$. The experiments in this work shows that with the best criterion MSRE, our model only introduced a stable deviation less than 8.62 iu based on the $\mathrm{C}_{7}-\mathrm{C}_{26}$ data when the carbon number was increased to $27-31$, which also a good demonstration of the interpolation and extrapolation fitting capability of the our method.

It has to be mentioned that the two dimension column were both operated isothermally in isothermal experiments and temperature programmed in temperature programmed experiments, in which the second dimension was always $+5{ }^{\circ} \mathrm{C}$ relative to the primary dimension. It also has to be mentioned that our method are proposed with an assumption that the separation in the second dimension column had been treated as in a pseudo-isothermal conditions in temperature programmed experiments because the length of the second column in $\mathrm{GC} \times \mathrm{GC} / \mathrm{TOF}-\mathrm{MS}$ is very short, i.e., usually ranging from $0.5 \mathrm{~m}$ to $2 \mathrm{~m}$. For example, the column used in our experiments is $1.8 \mathrm{~m}$ in length. However, it is not a real isothermal condition, and the accuracy of 
our method should be reassessed if the column is much longer than the configuration in our experiments.

To further evaluate our proposed method, the retention indices of 47 compounds identified from the MegaMix samples in $\mathrm{GC} \times \mathrm{GC} / \mathrm{TOF}-\mathrm{MS}$ temperature programmed experiments were also analyzed. For the 47 identified compounds, the small values of

$S T D$ and $\overline{S T D}$ of the converted retention index demonstrated that our model can be applied to analyze complex samples by calculating the retention index values of each compound in the second dimension column with an acceptable accuracy. Comparing with current methods, our regression model can get more accurate second dimension retention index. Moreover, fewer parameters have to be determined in the fitting function makes our model more practical.

\section{Acknowledgements}

This work was supported by the National Science Foundation of China (Nos. 61472282, 61300058 and 61271098) and Anhui Provincial Natural Science Foundation (No.1508085MF129).

\section{Appendix A. Supplementary data}

The following information can be found in the supplementary file: Figure S1, Figure S2, and Table S1

\section{References}


[1] G.J. Patti, O. Yanes, G. Siuzdak, Innovation: metabolomics: the apogee of the omics trilogy, Nat. Rev. Mol. Cell Biol, 13 (2012) 263-269.

[2] Z. Liu, J.B. Phillips, Comprehensive Two-Dimensional Gas Chromatography using an On-Column Thermal Modulator Interface, Journal of Chromatographic Science, 29 (1991).

[3] M. Adahchour, J. Beens, R.J.J. Vreuls, U.A.T. Brinkman, Recent developments in comprehensive two-dimensional gas chromatography $(\mathrm{GC} \times \mathrm{GC}):$ I. Introduction and instrumental set-up, Trends Anal. Chem., 25 (2006) 438-454.

[4] C. Oh, X. Huang, F.E. Regnier, C. Buck, X. Zhang, Comprehensive two-dimensional gas chromatography/time-of-flight mass spectrometry peak sorting algorithm, J. Chromatogr. A, 1179 (2008).

[5] B. Wang, A.Q. Fang, J. Heim, B. Bogdanov, S. Pugh, M. Libardoni, X.A. Zhang, DISCO: distance and spectrum correlation optimization alignment for two-dimensional gas chromatography time-of-flight mass spectrometry-based metabolomics, Anal. Chem., 82 (2010) 5069-5081.

[6] R.J. Western, P.J. Marriott, Retention correlation maps in comprehensive two-dimensional gas chromatography, J. Sep. Sci., 25 (2002) 832-838.

[7] M. Adahchour, J. Beens, R.J.J. Vreuls, U.A.T. Brinkman, Recent developments in comprehensive two-dimensional gas chromatography $(\mathrm{GC} \times \mathrm{GC})$ II. Modulation and detection, Trends Anal. Chem., 25 (2006).

[8] M. Adahchour, J. Beens, U.A.T. Brinkman, Recent developments in the application of comprehensive two-dimensional gas chromatography, J. Chromatogr. A, 1186 (2008).

[9] M. Adahchour, J. Beens, R.J.J. Vreuls, U.A.T. Brinkman, Recent developments in comprehensive two-dimensional gas chromatography $(\mathrm{GC} \times \mathrm{GC}):$ III. Applications for petrochemicals and 
organohalogens, Trends Anal. Chem., 25 (2006).

[10] J.F. Focant, A. Sjödin, W.E. Turner, D.G. Patterson, Measurement of selected polybrominated diphenyl ethers, polybrominated and polychlorinated biphenyls, and organochlorine pesticides in human serum and milk using comprehensive two-dimensional gas chromatography isotope dilution time-of-flight mass spectrometry, Anal. Chem., 76 (2004).

[11] B. Guthery, T. Bassindale, A. Bassindale, C.T. Pillinger, G.H. Morgan, Qualitative drug analysis of hair extracts by comprehensive two-dimensional gas chromatography/time-of-flight mass spectrometry, J. Chromatogr. A, 1217 (2010).

[12] R.E. Mohler, K.M. Dombek, J.C. Hoggard, E.T. Young, R.E. Synovec, Comprehensive Two-Dimensional Gas Chromatography Time-of-Flight Mass Spectrometry Analysis of Metabolites in Fermenting and Respiring Yeast Cells, Anal. Chem., 78 (2006).

[13] L. Mondello, P.Q. Tranchida, P. Dugo, G. Dugo, Comprehensive two-dimensional gas chromatography-mass spectrometry: A review, Mass Spectr. Rev., 27 (2008).

[14] F. Hufsky, K. Scheubert, S. Böcker, Computational mass spectrometry for small-molecule fragmentation, Trends in Analytical Chemistry, 53 (2014) 41-48.

[15] S.E. Stein, Retention Indices in NIST Chemistry WebBook, in, NIST Standard Reference Database Number 69, 2008.

[16] S. Stein, Mass Spectral Reference Libraries: An Ever-Expanding Resource for Chemical Identification, Anal. Chem., 84 (2012) 7274-7282.

[17] F. Rasche, K. Scheubert, F. Hufsky, T. Zichner, M. Kai, A. Svatoš, S. Böcker, Identifying the Unknowns by Aligning Fragmentation Trees, Anal. Chem., 84 (2012) 3417-3426.

[18] M.-F. Hérent, V.D. Bie, B. Tilquin, Determination of new retention indices for quick identification 
of essential oils compounds, J. Pharmaceut. Biomed., 43 (2007).

[19] L. Mondello, A. Salvatore, P.Q. Tranchida, A. Casilli, P. Dugo, G. Dugo, Reliable identification of pesticides using linear retention indices as an active tool in gas chromatographic-mass spectrometric analysis, Journal of Chromatography A, 1186 (2008).

[20] V.I. Babushok, P.J. Linstrom, J.J. Reed, I.G. Zenkevich, R.L. Brown, W.G. Mallard, S.E. Stein, Development of a database of gas chromatographic retention properties of organic compounds, J. Chromatogr. A, 1157 (2007) 414-421.

[21] R. Richmond, Database of structures and their gas chromatography retention indices, tagged with individual search windows, J. Chromatogr. A, 758 (1997).

[22] F. Bianchi, M. Careri, A. Mangia, M. Musci, Retention indices in the analysis of food aroma volatile compounds in temperature-programmed gas chromatography: Database creation and evaluation of precision and robustness, J. Sep. Sci, 30 (2007).

[23] T. Kind, G. Wohlgemuth, D.Y. Lee, Y. Lu, M. Palazoglu, S. Shahbaz, O. Fiehn, FiehnLib: Mass Spectral and Retention Index Libraries for Metabolomics Based on Quadrupole and Time-of-Flight Gas Chromatography/Mass Spectrometry, Anal. Chem., 81 (2009).

[24] J. Beens, R. Tijssen, J. Blomberg, Comprehensive Two-Dimensional Gas Chromatography (GC×GC) as a Diagnostic Tool, J. High Res. Chromatogr., 21 (1998).

[25] J. Beens, R. Tijssen, J. Blomberg, Prediction of comprehensive two-dimensional gas Chromatographic separations: A theoretical and practical Exercise, J. Chromatogr. A, 822 (1998) 233-251.

[26] R.J. Western, P.J. Marriott, Methods for generating second dimension retention index data in comprehensive two-dimensional gas chromatography, J. Chromatogr. A, 1019 (2003) 3-14. 
[27] S.K. Zhu, X. Lu, Y.Q. Qiu, T. Pang, H.W. Kong, C.Y. Wu, G.W. Xu, Determination of retention indices in constant inlet pressure mode and conversion among different column temperature conditions in comprehensive two-dimensional gas chromatography, J. Chromatogr. A, 1150 (2007) 28-36.

[28] T. Pang, S. Zhu, X. Lu, G.W. Xu, Identification of unknown compounds on the basis of retention index data in comprehensive two-dimensional gas chromatography, Journal of separation science, J. Sep. Sci., 30 (2007) 868-874.

[29] S. Bieri, P.J. Marriott, Generating Multiple Independent Retention Index Data in Dual-secondary Column Comprehensive Two-dimensional Gas Chromatography, Anal. Chem., 78 (2006).

[30] S. Bieri, P.J. Marriott, Dual-Injection System with Multiple Injections for Determining Bidimensional Retention Indexes in Comprehensive Two-Dimensional Gas Chromatography, Anal. Chem., 80 (2008).

[31] Y. Zhao, J. Zhang, B. Wang, S.H. Kim, A. Fang, B. Bogdanov, Z. Zhou, C. McClain, X. Zhang, A method of calculating the second dimension retention index in comprehensive two-dimensional gas chromatography time-of-flight mass spectrometry, J. Chromatogr. A, 1218 (2011) 2577-2583.

[32] C.v. Mühlen, P.J. Marriott, Retention indices in comprehensive two-dimensional gas chromatography, Anal. Bioanal. Chem., 401 (2011) 2351-2360.

[33] I. Koo, Y. Zhao, J. Zhang, S. Kim, X. Zhang, A method of calculating the second dimension hold-up time for comprehensive two-dimensional gas chromatography, Journal of Chromatography A, 1260 (2012) 193-199.

[34] E. Kováts, Gas-chromatographische Charakterisierung organischer Verbindungen. Teil 1: Retentionsindices aliphatischer Halogenide, Alkohole, Aldehyde und Ketone, Helv. Chim. Acta., 41 (1958). 
[35] J.E. Quintanilla-Lopez, R. Lebron-Aguilar, J.A. Garcia-Dominguez, The hold-up time in gas chromatography II. Validation of the estimation based on the concept of a zero carbon atoms alkane, Journal of Chromatography A, 767 (1997) 127-136.

[36] D.M. Bates, D.G. Watts, Nonlinear Regression Analysis and Its Application, 1997.

[37] R.O. Duda, P.E. Hart, D.G. Stork, Pattern Classification Second Edition, 2000. 


\section{Figure captions}

Fig. 1. Distribution of $\left\{a\left({ }^{2} T_{e}\right)\right\}$ and a nonlinear fitting curve. Circles represent $\left\{a\left({ }^{2} T_{e}\right)\right\}$ and solid line represents a fitting function.

Fig. 2. Distribution of $\left\{b\left({ }^{2} T_{e}\right)\right\}$ and a linear fitting curve. Circles represent $\left\{b\left({ }^{2} T_{e}\right)\right\}$; line represents a linear fitting function.

Fig. 3. Distribution of $\left\{b\left({ }^{2} T_{e}\right)\right\}$ and a nonlinear fitting curve. Circles represent adjusted $\left\{b\left({ }^{2} T_{e}\right)\right\}$ and blue curve is the fitting curve of function Eq. (15).

Fig. 4. The adjusted second dimension retention time map of the $C_{n}$-alkanes $\left(C_{7}-C_{31}\right.$ from left to right) constructed with the isothermal experimental data. The proposed ${ }^{2} t_{R^{-}}{ }^{2} T_{e}$ function with initial value of coefficients was used to fit the retention time values of all $\mathrm{C}_{\mathrm{n}}$-alkanes. The circles are the adjusted retention time of $\mathrm{C}_{\mathrm{n}}$-alkanes at different temperatures and the solid lines are fitting curves.

Fig. 5. The adjusted second dimension retention time map of the $n$-alkanes constructed from (a) the full isothermal experimental data; (b) $\mathrm{C}_{7}-\mathrm{C}_{26}$ data. The proposed ${ }^{2} t_{R^{-}}{ }^{2} T_{e}$ function and the criterion function MSE Eq. (4) was used to fit the retention time values of the $n$-alkanes. The solid lines depict the fitting curves and the circles depict the experimental data. 


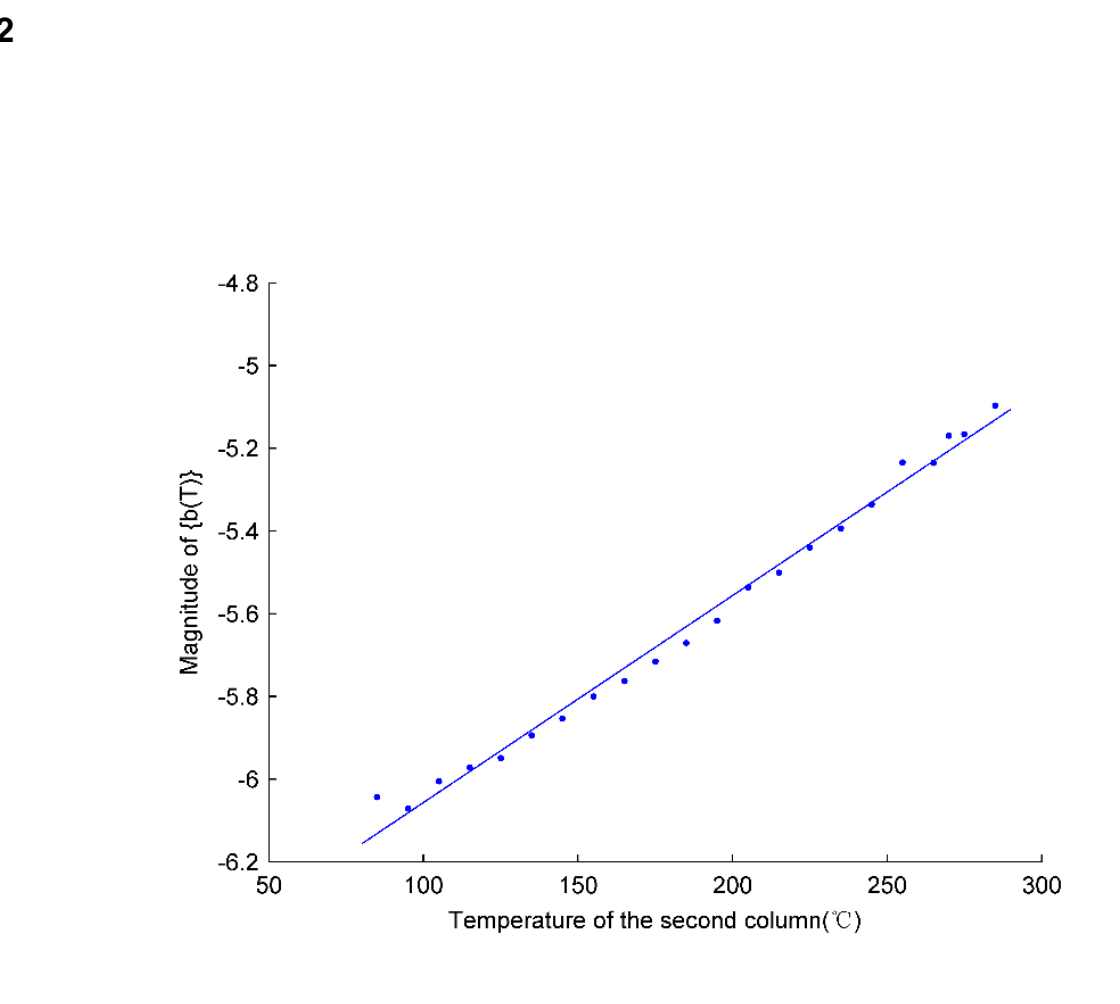

Figure 2

\section{Fure 2}

-

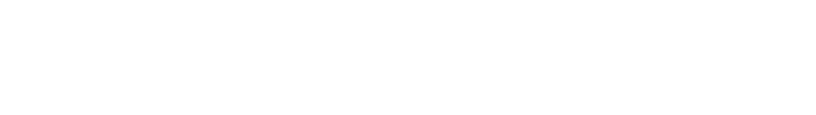
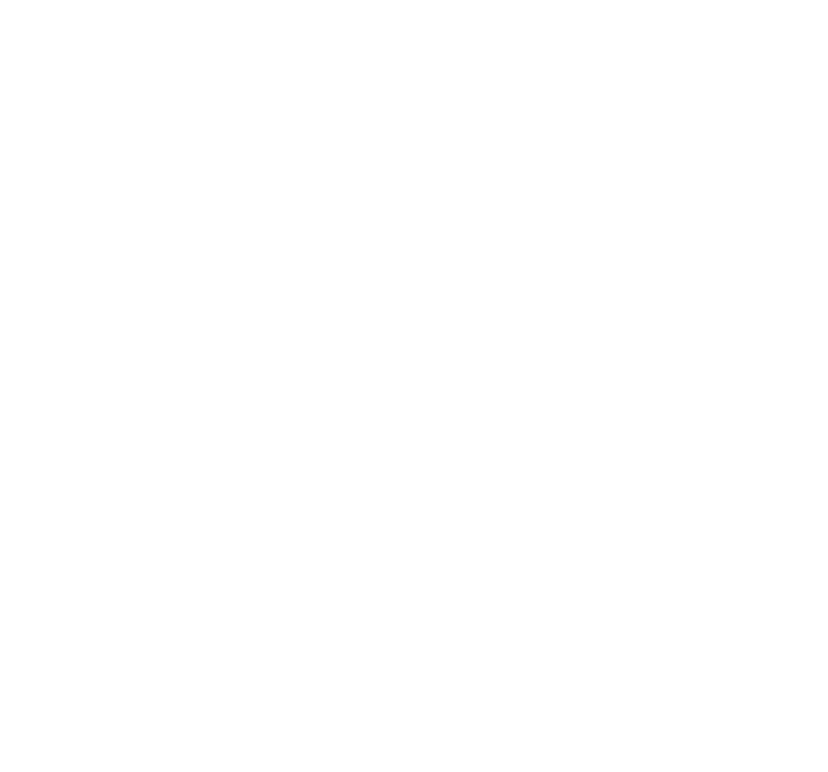

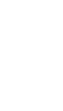
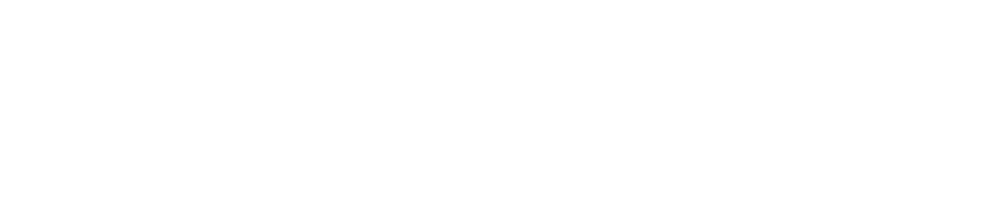


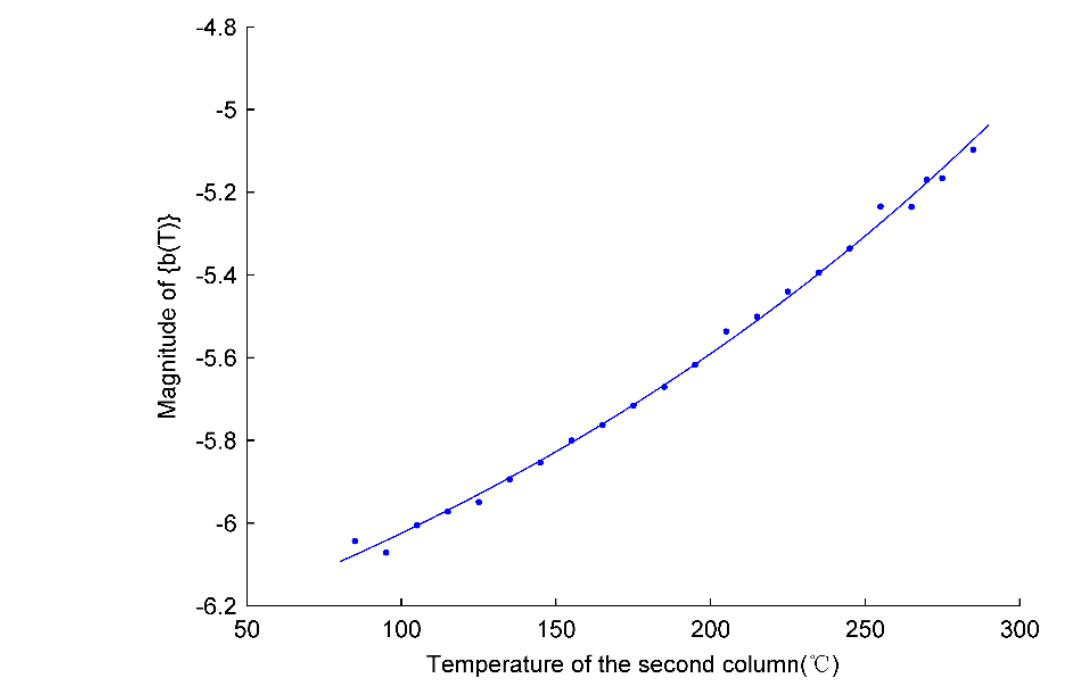

Figure 3 
Figure 4

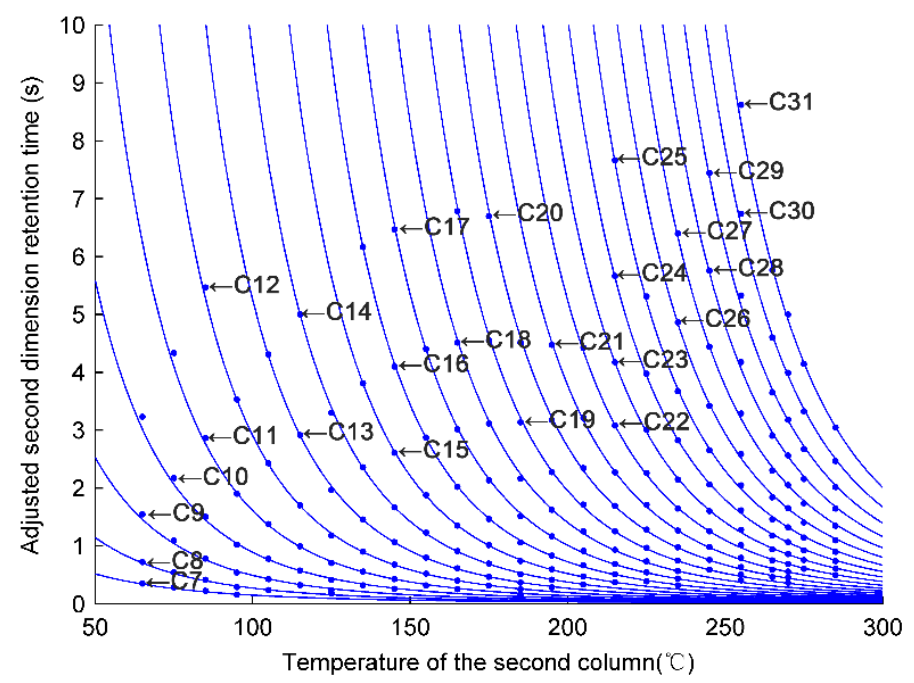


Figure 5
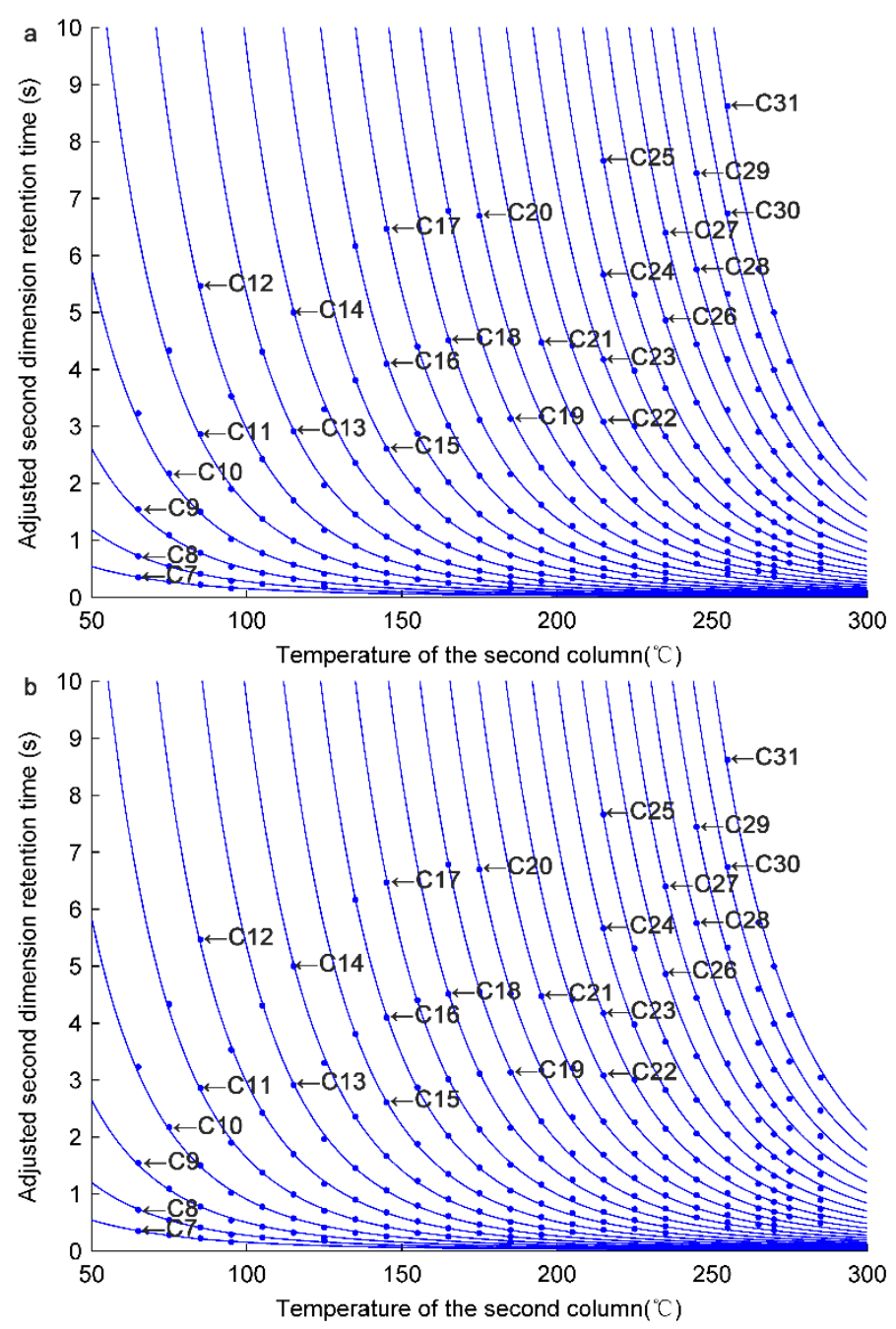
Table 1 The MAE, RMSE and MRE of all $n$-alkanes' $\left(\mathrm{C}_{7}-\mathrm{C}_{31}\right)$ retention time in the second dimension between the experimental and predicted values calculated by the proposed ${ }^{2} t_{R^{-}}{ }^{2} T_{e}$ function and different criteria.

\begin{tabular}{lccc}
\hline & MAE & RMSE & MRE \\
\hline Initial Value & 0.0303 & 0.0504 & 0.0205 \\
MSE & 0.0303 & 0.0429 & 0.0220 \\
MSRE & 0.0315 & 0.0495 & 0.0206 \\
Combination & 0.0302 & 0.0446 & 0.0207 \\
\hline
\end{tabular}


Table 2 The MAE and RMSE of all $n$-alkanes' $\left(\mathrm{C}_{7}-\mathrm{C}_{31}\right)$ retention index in the second dimension with the proposed ${ }^{2} t_{R^{-}}{ }^{2} T_{e}$ function and different criteria.

\begin{tabular}{lcc}
\hline & MAE & RMSE \\
\hline Initial Value & 6.17 & 8.80 \\
MSE & 6.91 & 10.24 \\
MSRE & 6.35 & 9.00 \\
Combination & 6.44 & 9.24 \\
\hline
\end{tabular}


Table $3 \mathrm{MAE}$ of ${ }^{2} I$ between the predicted and standard values from $\mathrm{C}_{27}$ to $\mathrm{C}_{31}$. The proposed ${ }^{2} t_{R^{-}}{ }^{2} T_{e}$ function was used. The parameters were calculated under different criteria with different parts of the isothermal experimental data $\left(\mathrm{C}_{7}-\mathrm{C}_{26}\right.$ or $\left.\mathrm{C}_{7}-\mathrm{C}_{31}\right)$.

\begin{tabular}{lccccc}
\hline & $\mathbf{C}_{\mathbf{2 7}}$ & $\mathbf{C}_{\mathbf{2 8}}$ & $\mathbf{C}_{\mathbf{2 9}}$ & $\mathbf{C}_{\mathbf{3 0}}$ & $\mathbf{C}_{\mathbf{3 1}}$ \\
\hline Initial Value & 7.11 & 7.38 & 6.87 & 6.88 & 6.21 \\
MSE $\left(\mathbf{C}_{\mathbf{7}}-\mathbf{C}_{\mathbf{2 6}}\right)$ & 12.44 & 13.25 & 13.17 & 13.79 & 12.52 \\
MSE $\left(\mathbf{C}_{\mathbf{7}}-\mathbf{C}_{\mathbf{3 1}}\right)$ & 7.09 & 7.57 & 7.69 & 8.15 & 7.88 \\
MSRE $\left(\mathbf{C}_{\mathbf{7}}-\mathbf{C}_{\mathbf{2 6}}\right)$ & 8.62 & 8.62 & 8.06 & 7.48 & 6.66 \\
MSRE $\left(\mathbf{C}_{\mathbf{7}}-\mathbf{C}_{\mathbf{3 1}}\right)$ & 7.82 & 8.03 & 7.49 & 7.33 & 6.60 \\
Comb $\left(\mathbf{C}_{\mathbf{7}}-\mathbf{C}_{\mathbf{2 6}}\right)$ & 9.20 & 9.18 & 9.12 & 8.92 & 8.22 \\
Comb $\left(\mathbf{C}_{\mathbf{7}}-\mathbf{C}_{\mathbf{3 1}}\right)$ & 7.52 & 7.76 & 7.48 & 7.88 & 7.35 \\
Zhao's $\left(\mathbf{C}_{\mathbf{7}}-\mathbf{C}_{\mathbf{2 6}}\right)$ & 9 & 10 & 13 & 21 & $\mathrm{NA}$ \\
\hline
\end{tabular}


Table 4 The MAE and RMSE of ${ }^{2} I$ with the proposed ${ }^{2} t_{R}{ }^{2}-T_{e}$ function and different criteria and four new datasets, while the full dataset had been taken as test set. The parameters were calculated under different criteria with different parts of the isothermal experimental data.

\begin{tabular}{c|lcc}
\hline \multicolumn{1}{l|}{ Dataset } & Parameter & MAE & RMSE \\
\hline \multirow{3}{*}{$\mathbf{D}_{\mathbf{1}}$} & MSE & 6.90 & 9.89 \\
& MSRE & 6.86 & 9.95 \\
& Comb & 6.93 & 9.97 \\
\hline \multirow{3}{*}{$\mathbf{D}_{\mathbf{2}}$} & MSE & 7.34 & 10.38 \\
& MSRE & 7.39 & 10.50 \\
& Comb & 7.24 & 10.27 \\
\hline \multirow{3}{*}{$\mathbf{D}_{\mathbf{3}}$} & MSE & 6.56 & 9.75 \\
& MSRE & 6.16 & 9.04 \\
& Comb & 6.20 & 9.06 \\
\hline \multirow{3}{*}{$\mathbf{D}_{\mathbf{4}}$} & MSE & 6.96 & 10.08 \\
& MSRE & 7.02 & 9.54 \\
& Comb & 6.79 & 9.32 \\
\hline
\end{tabular}

